\title{
Resolution of the VESUVIO spectrometer for High-energy Inelastic Neutron Scattering experiments
}

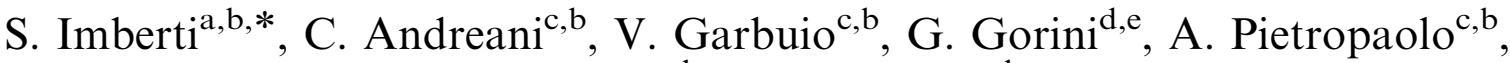 \\ R. Senesi ${ }^{\mathrm{c}, \mathrm{b}}, \mathrm{M}$. Tardocchi ${ }^{\mathrm{d}, \mathrm{e}}$ \\ a Università degli Studi di Roma Tre, Dipartimento di Fisica “E.Amaldi”, Roma, Italy \\ ${ }^{\mathrm{b}}$ CNR-INFM, Roma, Italy \\ ${ }^{\mathrm{c}}$ Università degli Studi di Roma Tor Vergata, Dipartimento di Fisica, Roma 60133, Italy \\ ${ }^{\mathrm{d}}$ Università degli Studi di Milano-Bicocca, Dipartimento di Fisica "G.Occhialini”, Milano, Italy \\ ${ }^{\mathrm{e}} \mathrm{CNR}$-INFM, Milano, Italy
}

Received 1 June 2005; accepted 30 June 2005

\begin{abstract}
New perspectives for epithermal neutron spectroscopy have been opened up as a result of the development of the Resonance Detector and its use on inverse geometry time-of-flight spectrometers at spallation sources. A special application of the Resonance Detector is the Very Low Angle Detector Bank (VLAD) for the VESUVIO spectrometer at ISIS, operating in the angular range $1^{\circ}<2 \theta<5^{\circ}$. This equipment allows High-energy Inelastic Neutron Scattering (HINS) measurements to be performed in the $(q, \omega)$ kinematical region at low wavevector $\left(q<10 \AA^{-1}\right)$ and high energy (unlimited) transfer $\hbar \omega>500 \mathrm{meV}$, a regime so far inaccessible to experimental studies on condensed matter systems. The HINS measurements complement the Deep Inelastic Neutron Scattering (DINS) measurements performed on VESUVIO in the high wavevector $q\left(20 \AA^{-1}<q<250 \AA^{-1}\right)$ and high energy transfer $(\hbar \omega>1 \mathrm{eV})$, where the short-time single-particle dynamics can be sampled. This paper will revise the main components of the resolution for HINS measurements of VESUVIO. Instrument performances and examples of applications for neutron scattering processes at high energy and at low wavevector transfer are discussed.
\end{abstract}

(C) 2005 Elsevier B.V. All rights reserved.

PACS: $28.20 . \mathrm{Cz} ; 29.30 . \mathrm{Hs} ; 78.70 . \mathrm{Nx}$

Keywords: Neutron; Scattering; Resolution; Epithermal

\footnotetext{
${ }^{*}$ Corresponding author. Current address: Università degli Studi di Roma Tor Vergata, Dipartimento di Fisica, Roma, Italy. Tel.: + 390672594429 ; fax: +39062023507.

E-mail address: silvia.imberti@roma2.infn.it (S. Imberti).
}

0168-9002/\$ - see front matter (C) 2005 Elsevier B.V. All rights reserved.

doi:10.1016/j.nima.2005.06.067 


\section{Introduction}

The advent of accelerator-based neutron sources has opened up new opportunities for epithermal $(\mathrm{eV})$ neutron spectroscopy and favored the development of novel instrumentation concepts in this area. In recent years experimental efforts have been devoted toward extending the dynamical range of neutron spectroscopy aiming to study: (a) the short-time single-particle (atoms or molecules) dynamics in quantum and molecular systems [1] and (b) the high-energy excitations in materials [2], i.e. electronic transitions in rare-earth metals and compounds [3], vibrational levels in insulators, semiconductors and magnetic materials [4].

In case (a) neutron spectroscopy at the $\mathrm{eV}$ energy is the unique experimental tool available to derive the single-particle momentum distribution, $n(p)$, and its second moment, the mean kinetic energy $\left\langle E_{k}\right\rangle$ [5]. These physical quantities are of great interest in those systems where the short-time dynamics departs from the classical behavior. The experimental technique is well established and known as Deep Inelastic Neutron Scattering (DINS), or Neutron Compton Scattering, and it is the analog of the Compton scattering of photons off electrons. DINS assumes that, at sufficiently high energy and owavevector transfers (typically $\hbar \omega>1 \mathrm{eV}, q>20 \AA^{-1}$ ), the scattering process is incoherent and occurs from free recoiling particles, both atoms and single molecules (this assumption is known as Impulse Approximation [6]). The study of the second class of phenomena requires neutron scattering processes with energy transfer in excess of about $500 \mathrm{meV}$ coupled to low wavevector transfer, typically $q<10 \AA^{-1}$. In this case both nuclear interactions and electromagnetic coupling of the neutron magnetic moment with the electron spin are relevant scattering processes. Low wavevector transfers are required due to the inherent decrease of the magnetic scattering intensity for increasing $q$ as the square of the magnetic form factor [29]. Nuclear and magnetic interactions provide access to forefront dynamical studies on high-lying vibrational states in molecular systems and high-energy excitations in magnetic systems and semiconductors, respectively. In recent years these studies have become possible thanks to the development of a novel technique, i.e. the High-energy Inelastic Neutron Scattering (HINS) [7], which has extended the dynamical range for $\mathrm{eV}$ neutron spectroscopy on VESUVIO.

Pioneering instrument developments in this dynamical range date back to the late 1970s, when Sinclair et al. [8] reported the general characteristics of an inverse geometry $\mathrm{eV}$ instrument exploiting the epithermal neutron flux on a pulsed neutron source. The adopted configuration was the Resonance Detector (RD), employing a metallic foil on the secondary flight path to define the energy of the scattered neutrons, via resonant neutron absorption, and a $\gamma$ detector to reveal the prompt cascade following the $(n, \gamma)$ reaction. After this original work, a few other instrument developments followed (see for example [9]); prototype instruments were constructed at the Harwell Linear Accelerator (UK) [10], at the Argonne National Laboratories (USA) [11] and at the ISIS pulsed neutron source of the Rutherford Appleton Laboratory (UK) [12].

At the time, large $\gamma$ detectors were employed and thus the signal-to-background ratio was severely limited by the heavy $\gamma$ and neutron background contamination typical of spallation sources, also demanding heavy shielding around the detectors. The RD concept was therefore soon abandoned in favor of the Resonance Filter (RF) configuration [13], which developed since the mid 1980s. The RF technique still employs an analyzer foil on the secondary flight path but in this case it is coupled with a neutron detector, e.g. ${ }^{3} \mathrm{He}$ or ${ }^{6} \mathrm{Li}$-based neutron counters, to reveal the neutrons nonabsorbed by the foil. The eVS spectrometer, installed at the ISIS facility [14], has been the first inverse geometry instrument routinely operating in the RF configuration for DINS measurements, in the range $1 \mathrm{eV}<\hbar \omega<20 \mathrm{eV}$ and $20 \AA^{-1}<q<250 \AA^{-1}$. Many DINS studies have been performed on a variety of systems. These include the single-particle dynamics of solid and fluid helium $\left({ }^{3} \mathrm{He}\right.$ and ${ }^{4} \mathrm{He}$ ) [15], noble gases in both solid and fluid phases [16], molecular systems [17], metals [18], fluid and solid hydrogen [19], glasses [20], graphite [21] and various metal hydrides [22]. 
Since the year 2002, the VESUVIO spectrometer upgraded the eVS instrument [23] expanding capability and scope of DINS measurements in the RF configuration. In addition, since the year 2003 VESUVIO has been equipped with a novel Very Low Angle Detector (VLAD) bank prototype at low scattering angles $\left(1^{\circ}-5^{\circ}\right)$, operating in the RD configuration, for HINS measurements. At present, HINS measurements on VESUVIO can be performed with a good detection efficiency up to $100 \mathrm{eV}$ [24]. The actual RD design is based on a combination of uranium or gold analyzer foils and solid-state [25] or scintillation photon counters [26].

This paper describes the resolution of the VESUVIO spectrometer in the RD configuration, in the $q$ and $\hbar \omega$ ranges of interest for typical HINS measurements. In Section 2 the main components of the VESUVIO spectrometer are revisited and the experimental setup of both RF and RD configurations are described. In Section 3 the main contributions to the energy and wavevector resolution are calculated as a function of the VLAD instrumental parameters. Performances attainable by further improvement of the instrument and a comparison with the resolution performances of some direct geometry chopper spectrometers are discussed. An example of an application is provided by recent HINS measurement performed on a sample of ice $\mathrm{I}_{h}$.

\section{Neutron spectroscopy at the $\mathrm{eV}$ energies with the VESUVIO Spectrometer}

\subsection{The instrument}

The VESUVIO spectrometer (see Fig. 1) is an inverse geometry instrument operating at the ISIS pulsed neutron source. The incident white neutron beam impinges on the sample located in a vacuum tank at a distance $L_{0}=11.055 \mathrm{~m}$ from the moderator. The final energy in the scattering process, for both DINS and HINS measurements, is defined by an analyzer foil, placed between the sample and the detector at about $L_{1}=0.5 \mathrm{~m}$ (DINS) or $2 \mathrm{~m}$ (HINS). For DINS measurements, the scattered neutrons, transmitted through the

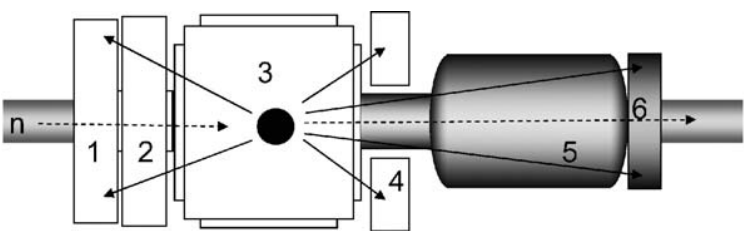

Fig. 1. A schematic view of the VESUVIO spectrometer; the neutrons $n$ are coming the moderator (not shown, on the left part of the picture) and impinge on the sample placed at the center of the vacuum chamber (3). In the DINS measurements the scattered neutrons are detected both by the forward scattering detectors (4) and by the high-resolution backscattering detectors (1). In the latter the analyzer foils (2) can also be cooled at Nitrogen temperature. On the right part of the picture the Very Low Angle Detector bank for HINS measurements is shown; it is composed by the cylindric vacuum chamber (5) and by the foils and the detectors bank (6).

foil, are recorded by ${ }^{6} \mathrm{Li}$ scintillators spanning the angular ranges $20^{\circ}<2 \theta<70^{\circ}$ and $120^{\circ}<2 \theta<160^{\circ}$. A special feature of DINS measurements is the high-resolution backscattering detectors bank: a proper vacuum chamber allocates analyzer foils of different thickness which can be cooled down through a closed cycle refrigerator.

Neutron scattering at low angle is performed through a cylindrical aluminum vacuum chamber, connected by a bellow to the VESUVIO sample chamber, allowing the neutrons to travel under vacuum for about $L_{1}=2 \mathrm{~m}$. The chamber is connected to the transmission beam tube and has a thin aluminum window $(2 \mathrm{~mm})$, enforced by six radial ribs $(3 \times 25 \mathrm{~mm})$. Next to the window there is a proper VLAD bank, with the analyzer foils stuck directly onto the detectors surface. The $\gamma$ rays following the resonant absorption are detected by YAP scintillators placed between $1^{\circ}<2 \theta<5^{\circ}$.

The pulsed nature of the source allows to reconstruct the whole kinematic of the scattering process by means of the time-of-flight technique. The total time of flight for a neutron travelling from moderator to detector is calculated through the following equation:

$t=\frac{L_{0}}{v_{0}}+\frac{L_{1}}{v_{1}}=A\left(\frac{L_{0}}{E_{0}^{1 / 2}}+\frac{L_{1}}{E_{1}^{1 / 2}}\right)$

where $L_{0}$ and $L_{1}$ are the primary and secondary flight paths, $v_{0}\left(E_{0}\right)$ and $v_{1}\left(E_{1}\right)$ represent the initial 
and final neutron velocities (energies), respectively, and $A=(m / 2)^{1 / 2}=72.3 \mathrm{eV}^{1 / 2} \times \mu s \times \mathrm{m}^{-1}, \quad m$ being the neutron mass.

The energy transferred in the scattering process is

$\hbar \omega=E_{0}-E_{1}$

while the momentum transferred is $\hbar q$ where $q$ is given by

$q=B\left(E_{0}+E_{1}-2 \sqrt{E_{0} E_{1}} \cos 2 \theta\right)^{1 / 2}$

with $B=\left(2 / \hbar^{2}\right)^{1 / 2}=21.97 \AA^{-1} \mathrm{eV}^{-1 / 2}$. For each scattering angle $2 \theta$, the value for $E_{1}$ is defined by the choice of the analyzer foil (see Table 2).

\subsection{The VESUVIO spectrometer operating in $R F$ and $R D$ configurations}

The experimental signal recorded in both RF and $\mathrm{RD}$ configurations is a time-of-flight spectrum where the expected count rate in a channel of width $\Delta t$ centered at time of flight $t$ is given by Ref. [32]:

$C(t) \Delta t=I\left(E_{0}\right) \frac{\mathrm{d} E_{0}}{\mathrm{~d} t} \Delta t N \frac{\mathrm{d}^{2} \sigma}{\mathrm{d} \Omega \mathrm{d} E_{1}} \eta \Delta \Omega \Delta E_{1}$

where $I\left(E_{0}\right)\left(\mathrm{d} E_{0} / \mathrm{d} t\right) \Delta t$ is the number of incident neutrons per square centimeter, $\mathrm{d}^{2} \sigma / \mathrm{d} \Omega \mathrm{d} E_{1}$ is the double differential scattering cross-section, $N$ is the number of scattering atoms, $\Delta \Omega$ is the detector solid angle, $\eta$ is the detection efficiency and $\Delta E_{1}$ is the energy resolution.

In the RF configuration the analyzer foil, located on the secondary flight path, selects the energy of the scattered neutrons by absorbing neutrons of energy close to a resonance energy $E_{1}$. Typical resonance energies in use are $4.91 \mathrm{eV}$ for the ${ }^{197} \mathrm{Au}$ foil and $6.67 \mathrm{eV}$ for the ${ }^{238} \mathrm{U}$ foil. The incident neutron energy is univocally determined from its time of flight (see Eq. (1)). In the RF configuration the term $\eta$ in Eq. (4) depends on $E_{1}$ and it is intrinsic to the counter, as the ${ }^{6} \mathrm{Li}$ neutron absorption cross-section depends on the inverse of the neutron velocity. The density of ${ }^{6} \mathrm{Li}$ atoms in the scintillation material, on the other hand, cannot be increased over a certain value, without producing a consistent loss in the light collection. Up to now, the best compromise allows to produce neutron detectors operating with satisfactory efficiency only below about $20 \mathrm{eV}$. The latter value can be regarded as an upper limit for the neutron energy accessible in the RF configuration. The range of wavevector and energy transfers which can be achieved for two typical final energies is illustrated in Fig. 2.

The experimental resolution for DINS measurements, as calculated in the early days of operation of the eVS spectrometer, can be found in the paper of Andreani et al. [33]. In this work are calculated

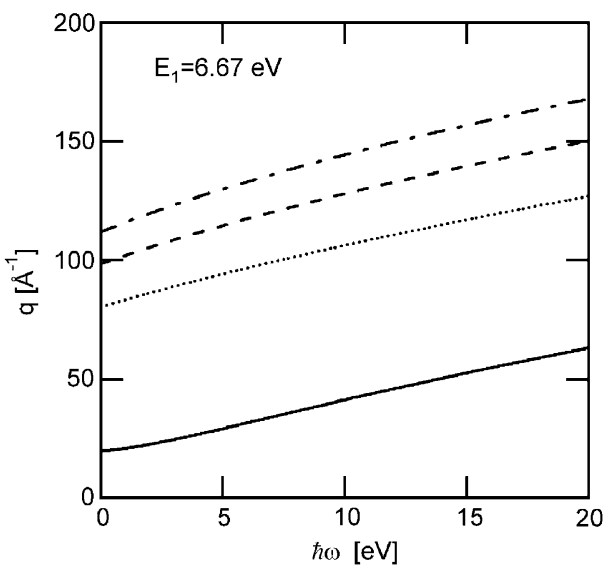

Fig. 2. Schematic plot illustrating the kinematical $(q, \hbar \omega)$ range for DINS in RF configuration, for $E_{1}=4.91 \mathrm{eV}$ (left) and $E_{1}=$ $6.67 \mathrm{eV}$ (right) and different values of scattering angles (from bottom to top: $20^{\circ}$ (full line), $90^{\circ}$ (dotted), $120^{\circ}$ (dashed) and $160^{\circ}$ (dash-dotted)). 
the expected resolution for the various components (energy, geometry and time of flight) and for different recoiling masses. This work has been recently extended by means of the Double Difference method, which allows to narrow the energy component of the resolution [34].

The RD configuration also uses an analyzer foil located on the secondary flight path to define the energy of the scattered neutrons. The foil resonantly captures neutrons over a narrow energy region and promptly emits radiative capture $\gamma$ and $\mathrm{X}$ rays. A photon detector measures the time at which the photons are detected, coincident on the time scale of interest with the time of absorbtion of the neutron in the foil. The best-suited isotopes are those whose cross-section shows well-isolated and intense peaks in the $1-100 \mathrm{eV}$ region with narrow widths (FWHM) as compared to the resonance energy. A list of suitable isotopes, achieving the above-mentioned characteristics, is reported in Table 2.

Fig. 3 shows the results of the calculation of the wavevector $q$ transfer as a function of $\hbar \omega$ at the scattering angle $2 \theta=1^{\circ}$ for five selected resonance energies. It can be observed that, at this scattering angle, energy transfers $\hbar \omega$ higher than $2 \mathrm{eV}$ coupled with $q$ lower than $10 \AA^{-1}$ can be accessed

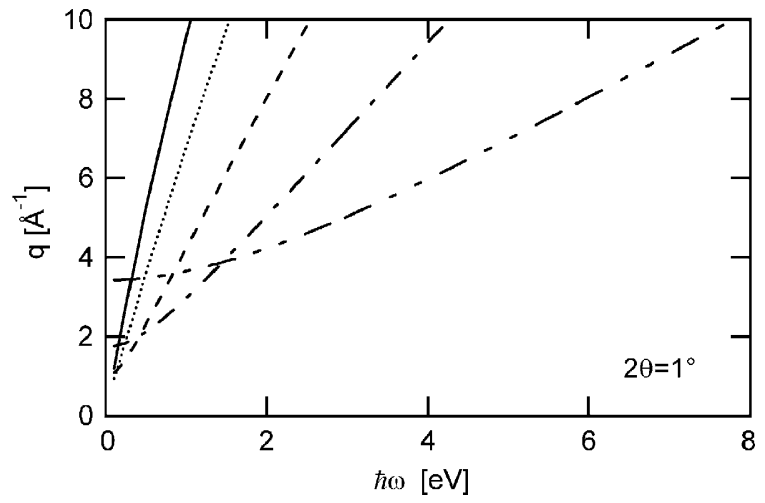

Fig. 3. Schematic plot illustrating the kinematical $(q, \hbar \omega)$ range for HINS in RD configuration at constant scattering angle $2 \theta=$ $1^{\circ}$ for different analyzer foils (see Table 2 for more details). From left to right: ${ }^{149} \mathrm{Sm}\left(E_{1}=0.872 \mathrm{eV}\right.$, full line $),{ }^{185} \mathrm{Re}$ $\left(E_{1}=2.16 \mathrm{eV}\right.$, dotted $),{ }^{238} \mathrm{U}\left(E_{1}=6.67 \mathrm{eV}\right.$, dashed $),{ }^{150} \mathrm{Sm}$ $\left(E_{1}=20.7 \mathrm{eV}\right.$, dash-dotted $)$ and ${ }^{168} \operatorname{Er}\left(E_{1}=79.7 \mathrm{eV}\right.$, dash-double dotted). by means of resonance energies $E_{1}$ higher than about $6 \mathrm{eV}$.

Fig. 4 shows $\hbar \omega$ and $q$ versus time of flight (tof) plotted for different values of scattering angle and final energy. In this figure the vertical lines plotted in each panel indicate the time of flight corresponding to a $6 \mathrm{eV}$ interband excitation in an insulator. In each figure the values of $\hbar \omega$ and $q$ are given by the intercepts of the vertical line with the corresponding curves for each angle. Due to the periodicity of the Bravais lattice, the wavevector can also be chosen outside the first Brillouin zone even if a lower value would correspond to higher scattering cross-sections [4]. In the example shown in the figure it appears that an energy excitation of $6 \mathrm{eV}$ can be observed at $q$ values below $10 \mathrm{~A}^{-1}$ for a scattering angle of $1^{\circ}$.

This requirement has guided the design and construction of the VLAD bank on the VESUVIO spectrometer. This device has been optimized for HINS measurements at the minimum angular range compatible with the hindrance of both the transmitted beam tube and detector's electronics, in order to access the smallest range of $q$ values.

\section{Resolution in $(\boldsymbol{q}, \omega)$ space}

The energy, $\Delta \hbar \omega$, and wavevector, $\Delta q$, resolution can both be written as a sum of different contributions associated with the uncertainties in the geometrical parameters $\left(L_{0}, L_{1}, 2 \theta\right)$, the time of flight $(t)$ and the selected final energy $\left(E_{1}\right)$. The resolution calculation is performed by considering $q$ and $\hbar \omega$ as a function of the experimental variables:

$\hbar \omega=\hbar \omega\left(t, E_{1}, L_{0}, L_{1}\right)$

$q=q\left(t, E_{1}, L_{0}, L_{1}, 2 \theta\right)$.

Let us denote the uncertainties on these experimental variables by $\Delta t, \Delta E_{1}, \Delta L_{0}, \Delta L_{1}, \Delta \theta$, and assume $[9,33,35]$ that they are independent of each other, gaussian distributed and sufficiently narrow for the differentials to remain constant over their range. 

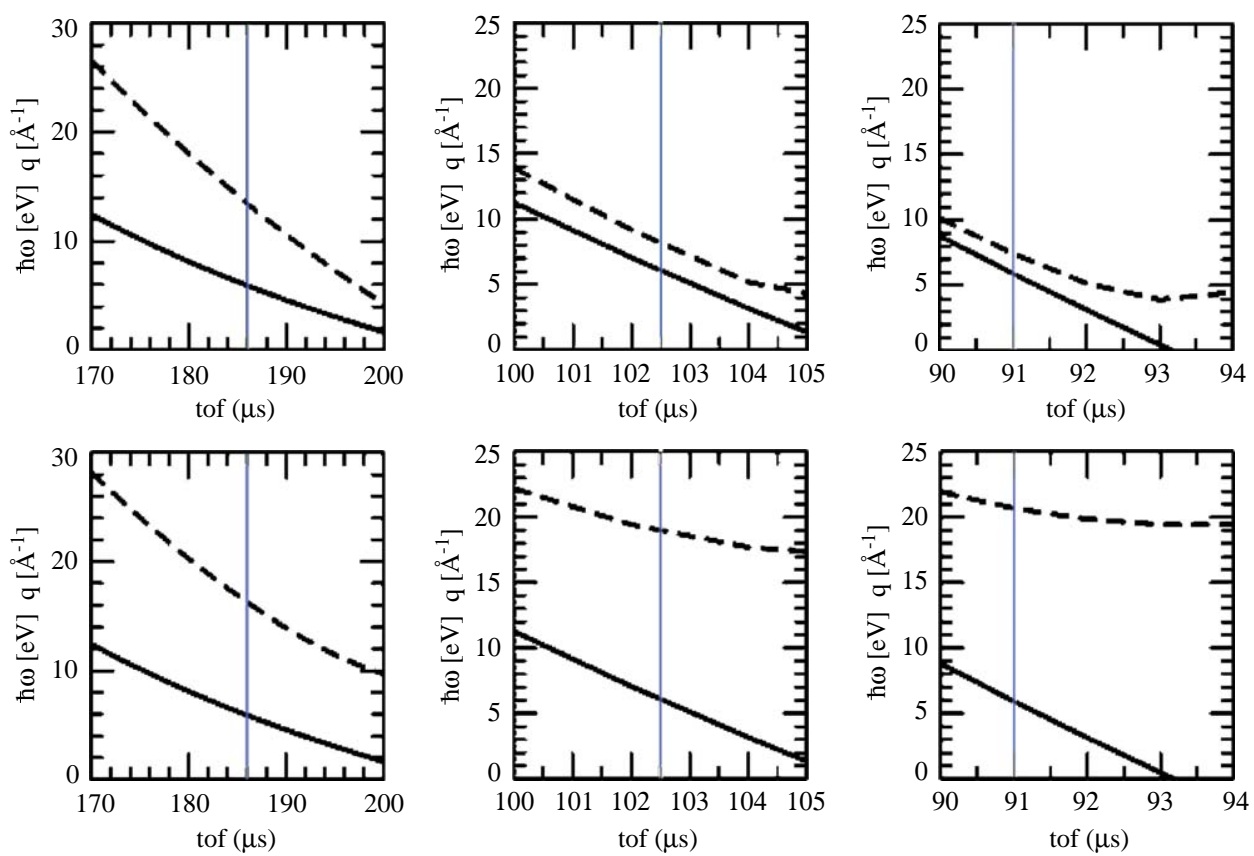

Fig. 4. Energy (full line) and wavevector transfers (dashed) as a function of the time of flight for two scattering angles $\left(1^{\circ}\right.$-top, $5^{\circ}$ bottom) and three different energy resonances of the foils $(20.9 \mathrm{eV}-\mathrm{left}, 79.7 \mathrm{eV}$-center, $102.6 \mathrm{eV}$-right). Vertical lines correspond to $\hbar \omega=6 \mathrm{eV}$, which is a typical interband excitation in an insulator.

The resulting formula yields:

$$
\begin{aligned}
(\Delta \hbar \omega)^{2}= & {\left[1+\left(\frac{E_{0}}{E_{1}}\right)^{3 / 2} \frac{L_{1}}{L_{0}}\right]^{2} \Delta^{2} E_{1}+\left[\frac{2 E_{0}^{3 / 2}}{A L_{0}}\right]^{2} \Delta^{2} t } \\
& +\left[\frac{2 E_{0}^{3 / 2}}{L_{0} E_{1}^{1 / 2}}\right]^{2} \Delta^{2} L_{1}+\left[\frac{2 E_{0}}{L_{0}}\right]^{2} \Delta^{2} L_{0} \\
(\Delta q)^{2}= & \left(\frac{B^{2}}{2 q}\right)^{2}\left[1-\left(\frac{E_{0}}{E_{1}}\right)^{3 / 2}\left(\frac{L_{1}}{L_{0}}\right)\right. \\
& \left.-\cos 2 \theta\left(\left(\frac{E_{0}}{E_{1}}\right)^{1 / 2}-\frac{L_{1}}{L_{0}} \frac{E_{0}}{E_{1}}\right)\right]^{2} \Delta^{2} E_{1} \\
& +\left(\frac{B^{2}}{2 q}\right)^{2}\left\{\left[\frac{2 E_{0}^{3 / 2}}{A L_{0}}\right]^{2} \Delta^{2} t+\left[\frac{2}{L_{0}} \frac{E_{0}^{3 / 2}}{E_{1}^{1 / 2}}\right]^{2} \Delta^{2} L_{1}\right. \\
& \left.+\left[\frac{2 E_{0}}{L_{0}}\right]^{2} \Delta^{2} L_{0}\right\}\left[\left(\frac{E_{1}}{E_{0}}\right)^{1 / 2} \cos 2 \theta-1\right]^{2} \\
& +\left(\frac{B^{2}}{2 q}\right)^{2}\left[2\left(E_{0} E_{1}\right)^{1 / 2} \sin 2 \theta\right]^{2} \Delta^{2} \theta
\end{aligned}
$$

\subsection{Energetic contribution}

The inherent finite width $\Delta E_{1}$ of the resonance energy determines the interval of the final energies $E_{1}$ accepted. The absorption resonance has an intrinsic width represented by the parameter $\Gamma$, the FWHM of the Lorentzian component, and a Doppler broadening, induced by the thermal motion of the lattice where the absorbing nucleus is embedded. In a solid of Debye temperature $\Theta_{\mathrm{D}}$ the Gaussian thermal broadening can be expressed by

$$
\Delta=\xi(T) E_{1}^{1 / 2} ; \quad \xi(T)=\sqrt{\frac{3}{2 M} m k_{\mathrm{B}} \Theta_{\mathrm{D}} \operatorname{coth}\left(\frac{3 \Theta_{\mathrm{D}}}{8 T}\right)}
$$

$k_{\mathrm{B}}$ being the Boltzmann constant and $T$ the absolute temperature, $\Delta$ is the half width at $1 / \mathrm{e}$ of a Gaussian shape while the corresponding FWHM is given by $\sqrt{\ln 2} \Delta \simeq 1.665 \Delta$. Overall, the convolution of both components results in a Voigt lineshape. 
The values for $\Delta E_{1}$ are listed in Table 2 for isotopes commonly used, or potentially useful, as analyzer foils, determined as the FWHM of the resonance peaks in the experimental neutron crosssection data at ambient temperature at Ref. [36]. A further enlargement factor should be introduced into the calculation by extracting the FWHM of the proper absorption profile $1-\mathrm{e}^{-\rho \sigma t}$, where $\rho$ is the number density of absorbing nuclei in the material, $\sigma\left(E_{1}\right)$ the scattering cross-section intensity and $t$ the thickness of the foil. Our calculation assumed a foil thickness $t$ smaller than $\frac{1}{\rho \sigma}$; in this manner the transmission function can be approximated with the $\sigma\left(E_{1}\right)$ itself [34].

\subsection{Angular contribution}

The finite dimensions of the detection device (composed of the analyzer foil and the $\gamma$ detector) and of the beam at the sample position give the main contributions to the angular uncertainty $\Delta \theta$. These dimensions, as shown in Fig. 5, define a minimum and a maximum scattering angle which correspond to a mean scattering angle $2 \theta_{m}$.

A bare geometrical calculation (see Fig. 5 for the meaning of the symbols) of $\Delta \theta=2 \theta_{\max }-2 \theta_{\min }$ provides

$\Delta \theta \sim\left(\frac{d_{\mathrm{D}}+d_{\mathrm{B}}}{L_{1}}\right)$

where $d_{\mathrm{B}}$ and $d_{\mathrm{D}}$ are the beam umbra plus penumbra and detector vertical dimension (diameter or side). This formula is a first-order

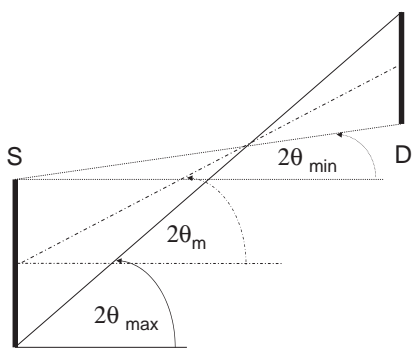

Fig. 5. Angular contribution to the $q$ resolution. Due to the finite size of the beam at the sample position and detector, we can find a minimum $2 \theta_{\min }$ and a maximum $2 \theta_{\max }$ scattering angle, being revealed at the same time bin relative to an average scattering angle $2 \theta_{m}$.
Table 1

Geometrical contributions to the resolution

\begin{tabular}{llllll}
\hline$L_{1}[\mathrm{~m}]$ & $\Delta L_{1}[\mathrm{~m}]$ & $L_{0}[\mathrm{~m}]$ & $\Delta L_{0}[\mathrm{~m}]$ & $2 \theta\left[^{\circ}\right]$ & $\Delta \theta\left[{ }^{\circ}\right]$ \\
\hline 2.00 & 0.005 & 11.055 & 0.02 & 2 & 1.43 \\
\hline
\end{tabular}

The choices are discussed in the text.

approximation valid for small angles and overestimates the uncertainty $\Delta \theta$ of at most $1 \%$ at the maximum angle considered here $\left(5^{\circ}\right)$. In this limit the angle uncertainty is independent of the scattering angle and it is proportional to the sum $d_{\mathrm{D}}+d_{\mathrm{B}}$ and inversely proportional to the secondary flight path $L_{1}$. The calculation for a $30 \mathrm{~mm}$ umbra plus penumbra diameter at the sample position and a $20 \mathrm{~mm}$ detector, corresponding to the actual configuration of VLAD, gives $\Delta \theta=$ $1.43^{\circ}$ at $2^{\circ}$ (Table 1 ).

\subsection{Flight path contributions}

The uncertainty on the primary flight path, $\Delta L_{0}$, is determined by the moderator depth and by the sample thickness. Neglecting the second contribution, which is one order of magnitude less than the first one, we can make the approximation $\Delta L_{0}=$ $2 \mathrm{~cm}$ [14]. The uncertainty on the secondary flight path, $\Delta L_{1}$, depends on the sample and detector thickness. As the latter in the RD configuration is given by the analyzer foil thickness, of the order of some tens of $\mu \mathrm{m}$, while the sample's one is assumed to be of $5 \mathrm{~mm}$, the analyzer's contribution can be neglected.

\subsection{Time-of-flight contribution}

The uncertainty on the time of flight is determined by three contributions [37]: the accelerator, the moderator and the data acquisition bin components. The time structure of the incident neutron beam is determined by the proton pulse time structure convoluted with the shape of the neutron pulse exiting the moderator. The proton bunch coming from the synchrotron operating at ISIS has a double structure in time, each bunch lasting $0.1 \mu$ s separated (peak to peak) by $0.23 \mu \mathrm{s}$. 
On the average the value of $\Delta t_{\mathrm{acc}}$ is assumed to be $0.3 \mu$ s. The neutron pulse shape coming from the water moderator at room temperature, $\Delta t_{\mathrm{mod}}$, is an energy-dependent term which is usually estimated by Monte Carlo simulation, assuming a $\delta(t)$ shape of the proton pulse. Following [37] one can calculate this time contribution and show that it decreases with increasing neutron energy, being $0.146 \mu$ s at $100 \mathrm{meV}$. The last contribution to the uncertainty comes from the time acquisition bin $\Delta t_{\text {bin }}=0.25 \mu \mathrm{s}$; this value is constant with the neutron energy. The sum in quadrature of the individual components yields:

$\Delta t=\left(\Delta t_{\mathrm{acc}}^{2}+\Delta t_{\mathrm{mod}}^{2}+\Delta t_{\mathrm{bin}}^{2}\right)^{1 / 2}=0.4 \mu \mathrm{s}$

\section{Results and discussion}

Table 2 shows the values of $\Delta \hbar \omega$ for several analyzers with values of the resonance energies $E_{1}$ between 1 and $100 \mathrm{eV}$. The resolution is almost constant as a function of $\hbar \omega$ for higher energy resonances, while it is a slightly increasing function of $\hbar \omega$ for low-energy resonances. Fig. 6 (left panel) shows the total resolution $\Delta \hbar \omega$ and the relative importance of the geometry, time and energy contributions as a function of $\hbar \omega$. It is evident that the resolution is indeed strongly dominated by the $\Delta E_{1}$ component. Fig. 6 (right panel) shows that an improvement of about $30 \%$ of $\Delta \hbar \omega$ can be achieved by cooling the analyzer foil to $77 \mathrm{~K}$, yielding a reduction of the Doppler broadening in the energetic contribution; a further improvement of $10 \%$ can be obtained by doubling the incident flight path. Fig. 7 shows the relative energy resolution as a function of the energy transfer for a few selected foils. The plot shows a decrease of the resolution down to a value of about $3 \%$, that is more rapid for lowenergy resonances and slower for high-energy resonances.

As far as the $q$ resolution is concerned, Fig. 8 shows the kinematic space $(q, \hbar \omega)$ for four resonances and the uncertainty $q \pm \frac{\Delta q}{2}$, indicated by the dashed lines. At low resonance energies, $\Delta q$ is lower than about $1 \AA^{-1}$ in the whole $\hbar \omega$ range,

Table 2

Resonance energy $E_{1}$ and widths $\Delta E_{1}$ for the different foils at room temperature, taken as FWHM from the experimental neutron scattering cross-section data available in Ref. [36]

\begin{tabular}{|c|c|c|c|c|c|c|c|}
\hline \multirow[b]{2}{*}{ Filter } & \multirow{2}{*}{$\begin{array}{r}E_{1} \\
(\mathrm{eV})\end{array}$} & \multirow{2}{*}{$\begin{array}{l}\Delta E_{1} \\
(\mathrm{meV})\end{array}$} & \multicolumn{5}{|l|}{$\Delta \hbar \omega(\mathrm{meV})$} \\
\hline & & & $\hbar \omega=10 \mathrm{meV}$ & $\hbar \omega=500 \mathrm{meV}$ & $\hbar \omega=3 \mathrm{eV}$ & $\hbar \omega=7 \mathrm{eV}$ & $\hbar \omega=20 \mathrm{eV}$ \\
\hline${ }^{149} \mathrm{Sm}$ & 0.872 & 83 & 98 & 113 & 224 & 492 & 1848 \\
\hline${ }^{240} \mathrm{Pu}$ & 1.06 & 56 & 66 & 74 & 133 & 272 & 965 \\
\hline${ }^{185} \mathrm{Re}$ & 2.16 & 58 & 69 & 73 & 99 & 157 & 429 \\
\hline${ }^{242} \mathrm{Pu}$ & 2.67 & 71 & 85 & 89 & 114 & 167 & 416 \\
\hline${ }^{197} \mathrm{Au}$ & 4.91 & 182 & 216 & 221 & 252 & 313 & 581 \\
\hline${ }^{238} \mathrm{U}$ & 6.67 & $103\left(66^{*}\right)$ & 125 & 128 & 144 & 174 & 307 \\
\hline${ }^{187} \mathrm{Os}$ & 12.7 & 100 & 135 & 138 & 151 & 177 & 286 \\
\hline${ }^{150} \mathrm{Sm}$ & 20.7 & 261 & 331 & 334 & 351 & 379 & 495 \\
\hline${ }^{238} \mathrm{U}$ & 20.9 & 177 & 243 & 246 & 262 & 290 & 404 \\
\hline${ }^{238} \mathrm{U}$ & 36.6 & 242 & 387 & 391 & 411 & 446 & 578 \\
\hline${ }^{238} \mathrm{U}$ & 66.0 & 320 & 701 & 707 & 736 & 784 & 952 \\
\hline${ }^{139} \mathrm{La}$ & 72.1 & 436 & 844 & 850 & 879 & 928 & 1098 \\
\hline${ }^{168} \mathrm{Er}$ & 79.7 & 120 & 785 & 791 & 826 & 883 & 1075 \\
\hline${ }^{238} \mathrm{U}$ & 102.6 & 410 & 1210 & 1217 & 1254 & 1315 & 1521 \\
\hline
\end{tabular}

$\Delta E_{1}$ represents the energy contribution to the instrument resolution. In the case of ${ }^{238} \mathrm{U}$ the value at a temperature $T=77 \mathrm{~K}$ is also reported, indicated with an $\left(^{*}\right)$. Columns 4-8 report the calculated resolution $\Delta \hbar \omega$ for selected values of the energy transfer $\hbar \omega$. 

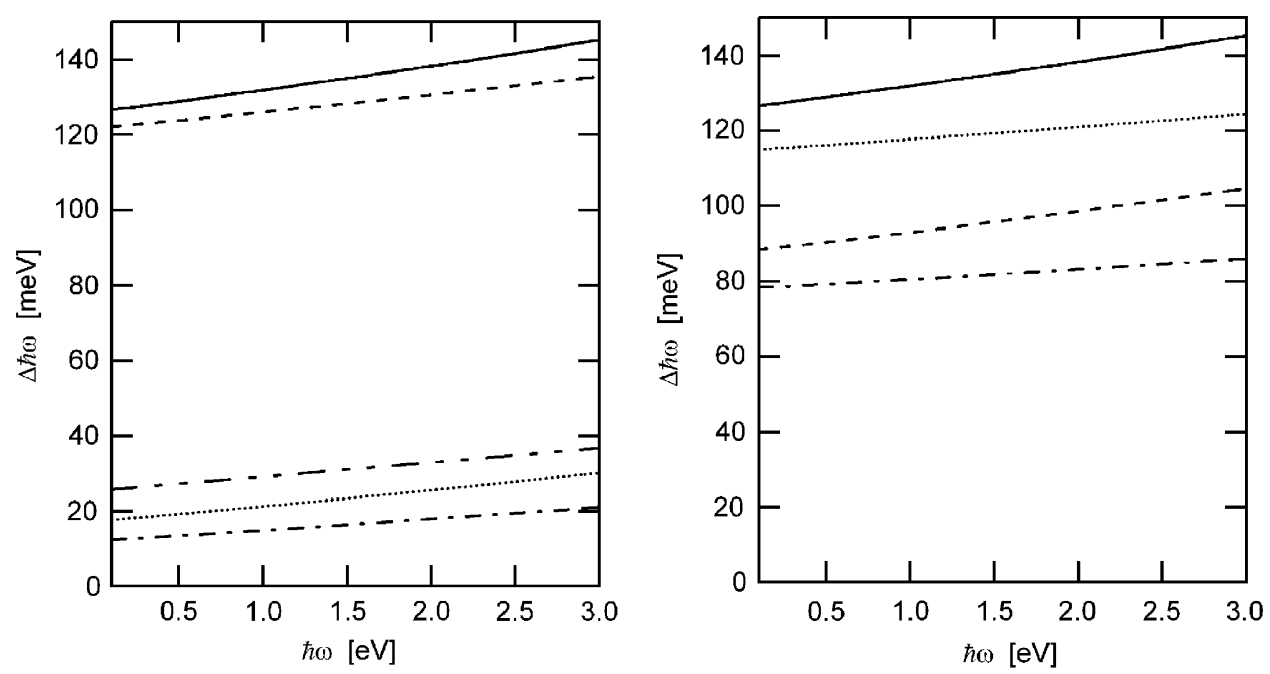

Fig. 6. Left: Total energy resolution $\Delta \hbar \omega$ (full line) for ${ }^{238} \mathrm{U}$ foil resonance at $6.67 \mathrm{eV}$ at $T=300 \mathrm{~K}$ with corresponding geometry ( $L_{0}=11.055 \mathrm{~m}$ dash-double dotted, $L_{1}=2 \mathrm{~m}$ dash-dotted), time (dotted) and energy (dashed) contributions. Right: Total energy resolution $\Delta \hbar \omega$ for: (a) ${ }^{238} \mathrm{U}$ resonance at $6.67 \mathrm{eV}$ at $300 \mathrm{~K}$ (full line); (b) ${ }^{238} \mathrm{U}$ resonance at $6.67 \mathrm{eV}$ at $T=77 \mathrm{~K}$ (dashed); (c) ${ }^{238} \mathrm{U}$ resonance at $6.67 \mathrm{eV}$ at $300 \mathrm{~K}$ and primary flight path $L_{0}=20 \mathrm{~m}$ (dotted); (d) a combination of (b) and (c) (dash-dotted).

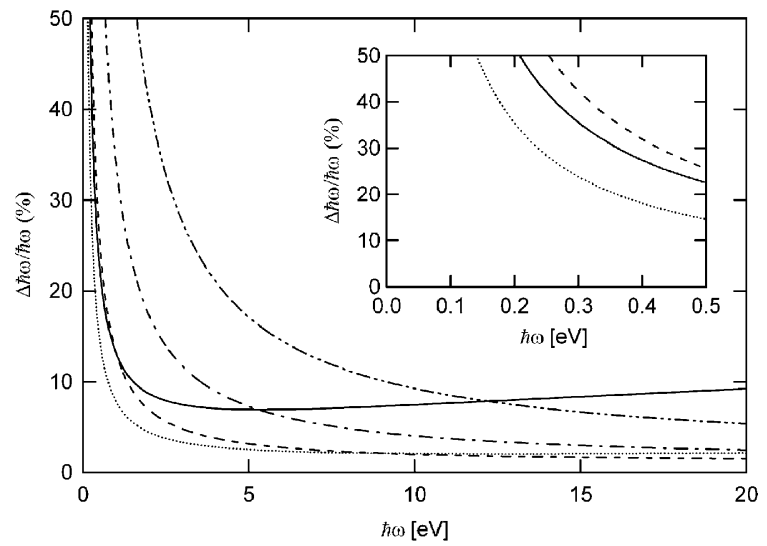

Fig. 7. Relative energy resolution as a function of the energy transfer. The different lines correspond to different analyzer foils (see Table 2 for details): ${ }^{149} \mathrm{Sm} E_{1}=0.872 \mathrm{eV}$ (full line), ${ }^{185} \mathrm{Re} E_{1}=2.16 \mathrm{eV}$ (dotted), ${ }^{238} \mathrm{U} E_{1}=6.67 \mathrm{eV}$ (dashed), ${ }^{150} \mathrm{Sm} \quad E_{1}=20.7 \mathrm{eV}$ (dash-dotted) and ${ }^{168} \mathrm{Er} \quad E_{1}=79.7 \mathrm{eV}$ (dash-double dotted). The first three resonances are also shown in the insert in the energy region $0-0.5 \mathrm{eV}$.

while for the resonance at $E_{1}=79.7 \mathrm{eV}$ it is less than $4 \AA^{-1}$ for $\hbar \omega>2 \mathrm{eV}$. The relative importance of the different contributions is detailed in Fig. 9, where the resonance width is shown to dominate the $q$ resolution too, except at the smallest energy transfers. Fig. 10 evidences instead the behavior of the $q$ resolution as a function of $q: \Delta q$ decreases by increasing $q$ and by decreasing the scattering angle. The $q$ resolution may be further improved by reducing the beam section at sample position and detector size, at the cost of the signal intensity, as shown in Fig. 10.

A summary of the results previously described is reported in Fig. 11. Points are calculated for selected values of the final energies, corresponding to some of the resonances listed in Table 2. The wavevector transfer and the relative resolution in $q$ and $\hbar \omega$ are plotted for two excitation energies, corresponding to two ideal experiments, performed on the $\mathrm{OH}$ stretching mode of ice $\left(\hbar \omega_{1}=\right.$ $417 \mathrm{meV}$ ) and an interband transition in an insulator $\left(\hbar \omega_{2}=6 \mathrm{eV}\right)$. The purpose of this figure is twofold: (1) to compare VESUVIO's performance in the $\hbar \omega<2 \mathrm{eV}$ regime with that of other instruments, specifically optimized in this range; (2) to show the capabilities of VESUVIO when the instrument operates for HINS experiment in a $(q, \hbar \omega)$ space so far unexplored. Fig. 11 shows that 

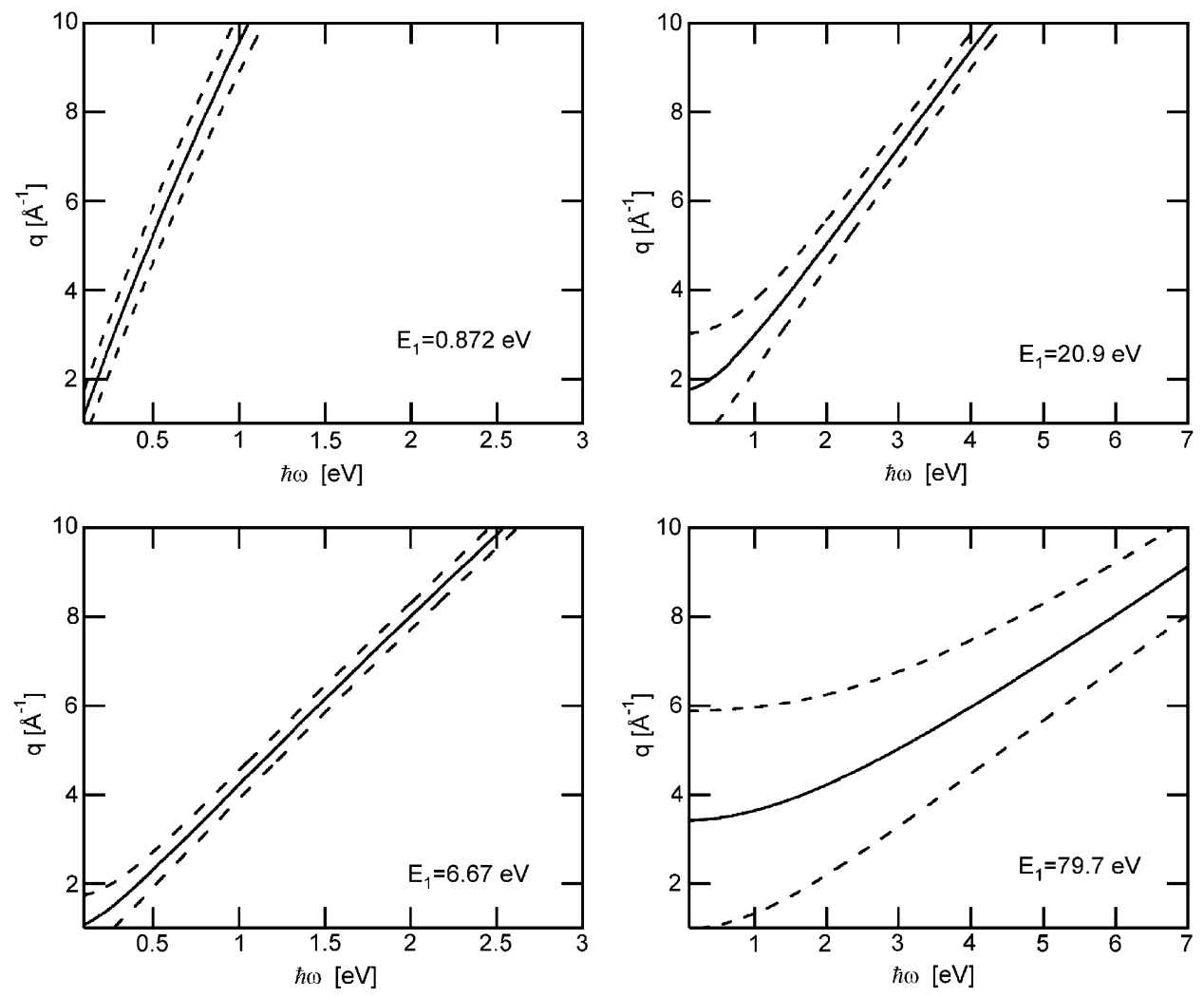

Fig. 8. Results for VLAD resolution in $(q, \hbar \omega)$ space for four different resonances at the scattering angle of $1^{\circ}$ : the full line represents the exchanged wavevector $q$, while the dashed line is $q \pm \frac{\Delta q}{2}$. From the upper left corner clockwise: ${ }^{149} \mathrm{Sm}$ at $0.872 \mathrm{eV},{ }^{238} \mathrm{U}$ at $20.9 \mathrm{eV}$, ${ }^{168} \mathrm{Er}$ at $79.7 \mathrm{eV}$ and ${ }^{238} \mathrm{U}$ at $6.67 \mathrm{eV}$.

the $q$ value diminishes at higher final energies: the left top panel shows that at $\hbar \omega_{1}=417 \mathrm{meV}$ wavevector lower than $10 \AA^{-1}$ values are available for every resonance energy $E_{1}$; the top right panel suggests the use of higher resonances $\left(E_{1}>60 \mathrm{eV}\right)$ if we wish to keep $q$ lower than $10 \AA^{-1}$ at $\hbar \omega_{2}=6 \mathrm{eV}$. Concerning the resolution requirements, given that the relative resolutions typically increase by increasing the final energy, it appears that best-suited foils are those with energy resonances in the range $0-4 \mathrm{eV}$ at $\hbar \omega_{1}=417 \mathrm{meV}$ (bottom-left panel) and $10-40 \mathrm{eV}$ at $\hbar \omega_{2}=6 \mathrm{eV}$ (bottom-right panel). This figure clearly shows that the VLAD bank of VESUVIO allows to perform HINS measurements, by selecting a suitable analyzer with optimized $q, \Delta \hbar \omega$ and $\Delta q$ values.

The VLAD equipment on VESUVIO allows to perform measurements that in the low-energy transfer range $(\hbar \omega<2 \mathrm{eV})$ are routinely performed on other instruments. Thus it is interesting to compare the resolution performance of VESUVIO with that available for instance on chopper instruments. Examples are HET [38], MARI [39] and MAPS [40] at ISIS or HRMECS at IPNS (Argonne National Lab., IL, USA) [41], with a fractional energy resolution at the elastic peak typically of $\Delta \hbar \omega / E_{0}=2-6 \%, 1-2 \%, 1-5 \%$, $2-4 \%$, respectively. This resolution can be considered an upper limit, with respect to the value at 


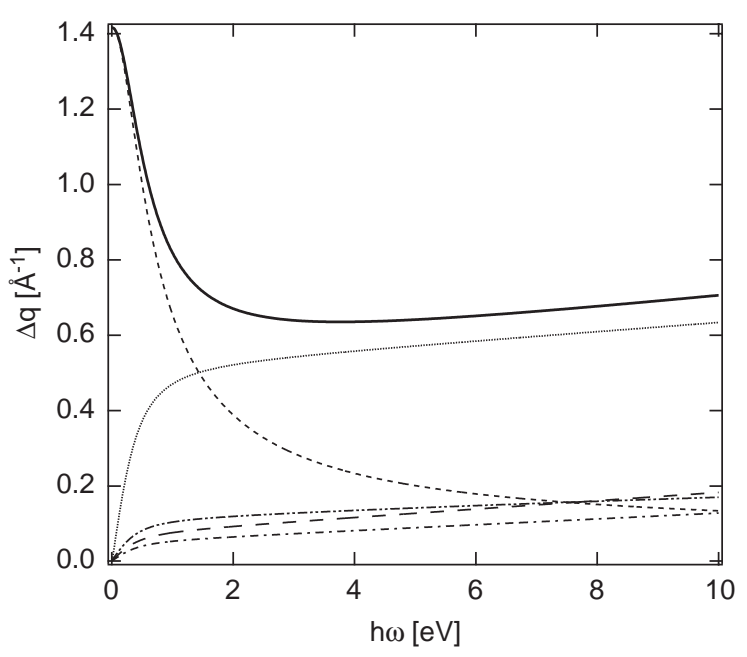

Fig. 9. Total $q$ resolution $\Delta q$ (full line) at $2 \theta=2^{\circ}$ for ${ }^{238} \mathrm{U}$ foil resonance at $6.67 \mathrm{eV}$ with corresponding components: (from top to bottom) energy (dotted), angle (dashed), primary flight path ( $L_{0}=11.055 \mathrm{~m}$ dash-double dotted) time (long dash) and secondary flight path $\left(L_{1}=2 \mathrm{~m}\right.$, dash-dotted) contributions.

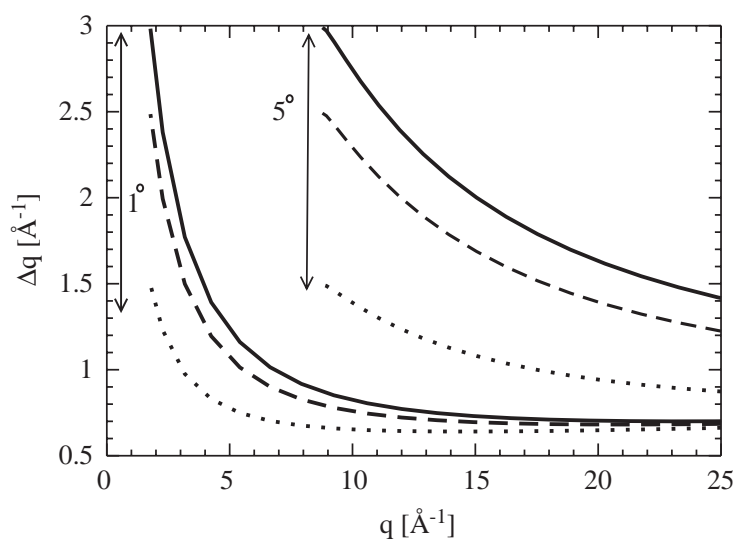

Fig. 10. The $q$ resolution, $\Delta q$, for different sizes of sample and detectors $d_{\mathrm{D}}+d_{\mathrm{B}}: 30 \mathrm{~mm}$ (full line), $25 \mathrm{~mm}$ (dashed), $15 \mathrm{~mm}$ (dotted), for two scattering angles and fixed final energy $\left(E_{1}=20.9 \mathrm{eV}\right)$.

finite energy transfer. Another interesting comparison can be carried out with the inverse geometry, high-resolution spectrometer TOSCA [42], provid- ing a resolution $\Delta \hbar \omega / \hbar \omega=1.5-3 \%$, but accessing much higher $q$ transfers. It is clear from the inset of Figs. 7 and 11 that the whole set of instruments provide better resolution than VESUVIO for energy transfers lower than about $2 \mathrm{eV}$. For example, as shown in Fig. 11 at the reference value $\hbar \omega=417 \mathrm{meV}$ the energy resolution calculation of the VLAD bank with the ${ }^{242} \mathrm{Pu}$ foil $\left(E_{1}=\right.$ $2.67 \mathrm{eV}$ ) yields $\Delta \hbar \omega / \hbar \omega=18 \%$. Nevertheless, a recent experiment [7] on a sample of ice $I_{h}$ carried out employing the prototype VLAD bank has shown the feasibility of HINS experiments for measuring the intramolecular vibrations in ice. The measurements have been performed using a $q$ range between 3 and $8 \mathrm{~A}^{-1}$ and with an energy resolution of about $140 \mathrm{meV}$ at $417 \mathrm{meV}$ $(\Delta \hbar \omega / \hbar \omega=30 \%)$. The density of states for ice compared quite well with that measured in a previous INS experiment performed on HRMECS [30].

It has to be stressed that the VLAD bank of VESUVIO gives a unique performance when higher energy excitations need to be measured. This is made possible by the unlimited energy transfer range available on an inverse geometry instrument. Fig. 11 shows as an example the resolution calculated for a $6 \mathrm{eV}$ interband transition in an insulator, employing the ${ }^{187}$ Os foil $\left(E_{1}=12.7 \mathrm{eV}\right)$, which allows $\Delta \hbar \omega / \hbar \omega=3 \%$.

In conclusion the VLAD bank allows to access a former unexplored high-energy transfer range $(\hbar \omega>1 \mathrm{eV})$, with a wavevector transfer tunable between 5 and $20 \AA^{-1}$ depending on the choice of the analyzer foil. The higher resonances $\left(E_{1}>20 \mathrm{eV}\right)$ allow to access the lowest $q$ values with a good energy resolution. These are also best suited for experiments on high-energy excitations and when $q$ resolution could be relaxed. Lowenergy resonances on the contrary have good resolution both in $\hbar \omega$ and $q$ and can therefore be successfully employed when the low $q$ transfer is not compelling, as in the measurement of interband transitions. VESUVIO operating in the RD configuration at a low scattering angle will enable high-energy inelastic neutron scattering studies in a wide $(q, \hbar \omega)$ range, which can be regarded as a necessary complement to the 

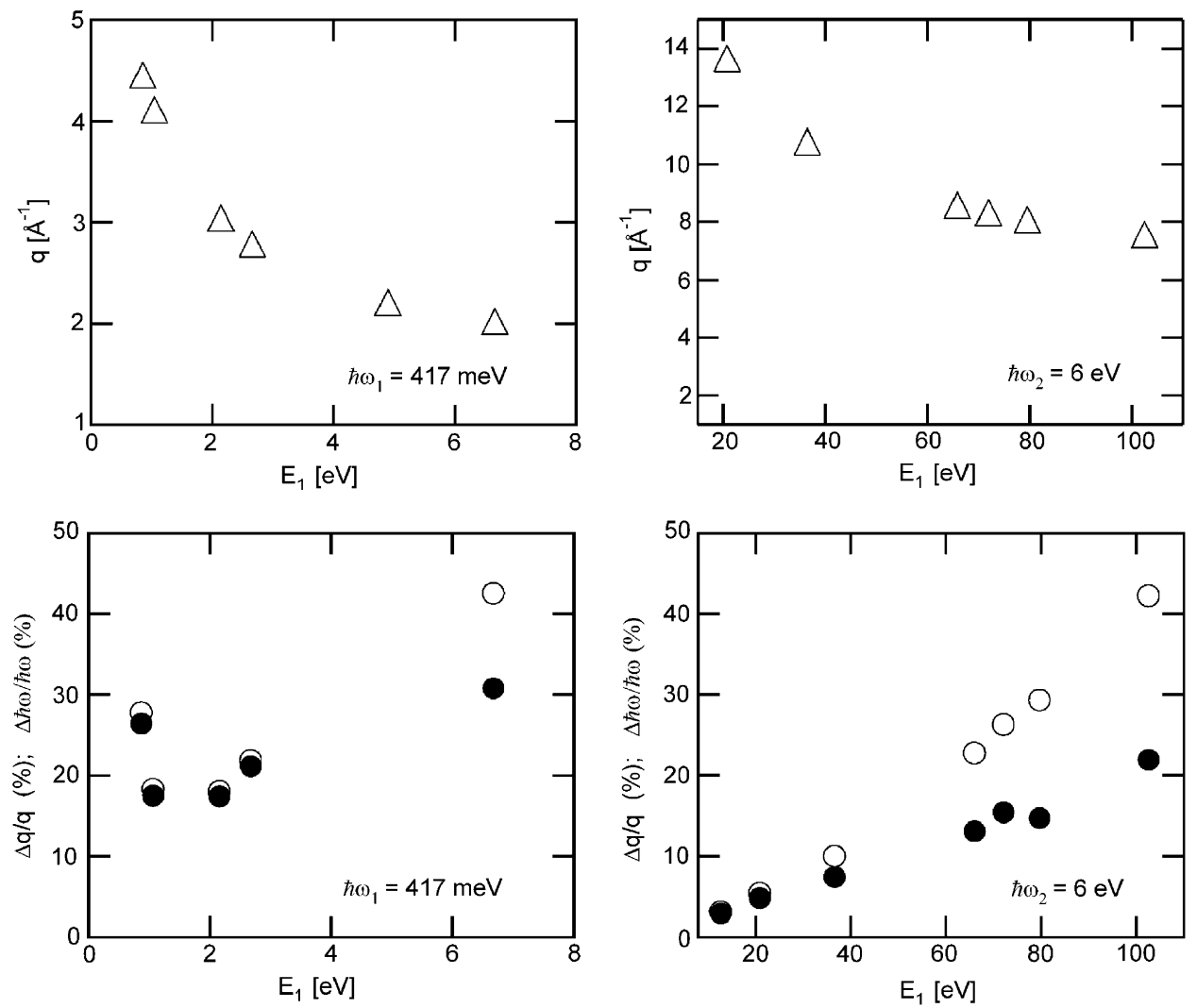

Fig. 11. Wavevector transfer $q(\triangle)$ and relative energy $(\bullet)$ and wavevector ( $\circ$ ) resolutions at fixed energy transfer and scattering angle $\left(1^{\circ}\right)$ as a function of final energy, selected by resonance absorption. The energy transfer is $\hbar \omega=417 \mathrm{meV}$ (left), corresponding to the $\mathrm{OH}$ stretch mode in water and $\hbar \omega=6 \mathrm{eV}$ (right), which corresponds to the energy interband excitation in an insulator. Note that the choice of the resonance energy allows to tune the performances of VLAD.

chopper spectrometers operating at spallation neutron sources.

\section{Acknowledgements}

The authors would like to thank: the ISIS engineering team and the RMP s.r.l. (Acilia, Roma); the ISIS sample environment group, a special mention for J. Dreyer, for help with sample rigs, cryogenics and temperature control; the electronic team, and N. Rhodes and E. Schooneveld for their support during the experiments on eVS and VESUVIO and the detector tests on the VLAD bank. We acknowledge the financial support of the European Community within the projects VESUVIO and e.VERDI of the program Access to Research Infrastructure Program in FP5 and FP6. We acknowledge the Consiglio Nazionale delle Ricerche (CNR)-Italy for financial support within the CNR-CCLRC agreement.

\section{References}

[1] P.C. Hohenberg, P.M. Platzmann, Phys. Rev. 152 (1966) 198 ;

G.K. Ivanov, Yu.S. Sayasov, Sov. Phys. Uspekhi 9 (1967) 670 (1966 Usp. Fiz. Nauk. 90 47).

[2] R.N. Silver, Allen 1984 Proc. Workshop on High Energy Excitations in Condensed Matter LA-10227-C. 
[3] R. Osborn, Argonne National Laboratory Report IL 60439, 1996;

A.D. Taylor, R. Osborn, K.A. McEwen, W.G. Stirling, Z.A. Bowden, W.G. Williams, E. Balcar, S.W. Lovesey, Phys. Rev. Lett. 61/11 (1988) 1309;

G. Amoretti, A. Blaise, R. Caciuffo, J.M. Fournier, M.T. Hutchings, R. Osborn, A.D. Taylor, Phys. Rev. B 40/3 (1989) 1856.

[4] T. Morgan, J.A. Blackman, J.F. Cooke, Phys. Rev. B 33/ 10 (1986) 7154;

J.F. Cooke, J.A. Blackman, Phys. Rev. B 26/8 (1982) 4410.

[5] P.E. Sokol, R.N. Silver, Momentum Distributions, Plenum Press, New York, 1989.

[6] S.W. Lovesey, Theory of Neutron Scattering from Condensed Matter, vol. 1, Oxford University Press, London, 1987.

[7] A. Andreani, G. Gorini, E. Perelli-Cippo, A. Pietropaolo, N.J. Rhodes, E.M. Schooneveld, R. Senesi, M. Tardocchi, Appl. Phys. Lett. 85 (2004) 5454.

[8] R.N. Sinclair, M.C. Moxon, J.M. Carpenter, Bull. Am. Phys. Soc. 22 (1977) 101.

[9] D.R. Allen, E.W.J. Mitchell, R.N. Sinclair, J. Phys. E: Sci. Instrum. 13 (1980) 639;

J.M. Carpenter, D.L. Price, N.J. Swanson, Argonne National Laboratory Report ANL-78-88 (1978);

J.M. Carpenter, T.H. Blewitt, D.L. Price, S.A. Werner, Phys. Today 42 (1979);

R.G. Johnson, C.D. Bowman, Phys. Rev. Lett. 49 (1982) 797.

[10] M.S. Coates, P.P. Thomas, B.P. Clear, R.N. Sinclair, J.E. Lynn, 1977, 4th Natl. Conf. on Neutron Physics (Kiev) vol. 4 p. 139.

[11] R.K. Crawford, Proc. ICANS IX-Int. Collab. on Advanced Neutron Sources 1986, p. 397.

[12] L.C.W. Hobbies, G.H. Rees, G.C. Stirling, Rutherford Laboratory Report RL-77-064/C, 1977.

[13] A.D. Taylor, R.A. Robinson, P.A. Seeger, Nucl. Instr. and Meth. 224 (1984) 133.

[14] J. Mayers, A.C. Evans, Rutherford and Appleton Laboratory RAL-91-048 (1991).

[15] M. Celli, M. Zoppi, J. Mayers, Phys. Rev. B 58 (1998) 242; R. Senesi, C. Andreani, D. Colognesi, A. Cunsolo, M. Nardone, Phys. Rev. Lett. 86 (2001) 4584.

[16] D.A. Peek, I. Fujita, M.C. Schmidt, R.O. Simmons, Phys. Rev. B 45 (1992) 9680;

M.A. Fradkin, S.X. Zeng, R.O. Simmons, Phys. Rev. 49 (1994) 3197;

D.A. Peek, M.C. Schmidt, I. Fujita, R.O. Simmons, Phys. Rev. B 45 (1992) 9671.

[17] C. Andreani, E. Degiorgi, R. Senesi, F. Cilloco, D. Colognesi, J. Mayers, M. Nardone, E. Pace, J. Chem. Phys. 114 (2001) 387;

C. Andreani, D. Colognesi, E. Degiorgi, M.A. Ricci, J. Chem. Phys. 115 (2001) 11243.

[18] S. Fulton, R.A. Cowley, A.C. Evans, J. Phys.: Condens. Matter 6 (1994) 2977;

A.C. Evans, J. Mayers, D.N. Timms, J. Phys.: Condens. Matter 6 (1994) 4197.
[19] W.E. Langel, D.L. Price, R.O. Simmons, P.E. Sokol, Phys. Rev. B 38 (1988) 275;

C. Andreani, D. Colognesi, E. Pace, Phys. Rev. B 60 (1999) 10008.

[20] F.J. Bermejo, F.J. Mompean, A. Srinivasan, J. Mayers, A.C. Evans, Phys. Lett. A 189 (1994) 333;

J. Dawidowski, F.J. Bermejo, L. Fernandez Barquin, P. Gorria, J.M. Barandarian, A.C. Evans, J. Mayers, Phys. Lett. A 214 (1996) 59.

[21] M.P. Pauli, R.S. Holt, J. Phys. C: Solid State Phys. 21 (1988) 3633;

A.L. Fielding, D.N. Timms, J. Mayers, Europhys. Lett. 44 (1998) 255.

[22] E.B. Karlsson, C.A. Chatzidimitriou-Dreismann, T. Abdul-Redah, R.M.F. Streffer, B. Hjörvarsson, J. Öhrmalm, J. Mayers, Europhys. Lett. 46 (1999) 617.

[23] R. Senesi, C. Andreani, Z. Bowden, D. Colognesi, E. Degiorgi, A.L. Fielding, J. Mayers, M. Nardone, J. Norris, M. Praitano, N.J. Rhodes, W.G. Stirling, J. Tomkinson, C. Uden, Physica B 276-278 (2000) 200;

A.L. Fielding, J. Mayers, Nucl. Instr. and Meth. A 480 (2002) 680.

[24] M. Tardocchi, A. Pietropaolo, C. Andreani, A. Bracco, A. D’Angelo, G. Gorini, S. Imberti, R. Senesi, N.J. Rhodes, E.M. Schooneveld, Nucl. Instr. and Meth. A 526 (2004) 477.

[25] C. Andreani, A. D'Angelo, G. Gorini, S. Imberti, A. Pietropaolo, N.J. Rhodes, E.M. Schooneveld, R. Senesi, M. Tardocchi, Appl. Phys. A 78 (2004) 903.

[26] A. Pietropaolo, C. Andreani, A. D'Angelo, G. Gorini, S. Imberti, N.J. Rhodes, E.M. Schooneveld, R. Senesi, M. Tardocchi, Proc. 11th Internat. Symposium Capture Gamma-Ray Spectroscopy (Prague), World Scientific, 2003.

[29] S.W. Lovesey, Theory of Neutron Scattering from Condensed Matter, vol. 2, Oxford University Press, London, 1987.

[30] C. Andreani, P. Bosi, F. Sacchetti, C.K. Loong, J. Chem. Phys. 83 (1985) 750 and references therein.

[32] C.G. Windsor, Pulsed Neutron Scattering, Taylor and Francis, London, 1981.

[33] C. Andreani, G. Baciocco, R.S. Holt, J. Mayers, Nucl. Instr. and Meth. A 276 (1989) 297.

[34] C. Andreani, D. Colognesi, E. Degiorgi, A. Filabozzi, M. Nardone, E. Pace, A. Pietropaolo, R. Senesi, Nucl. Instr. and Meth. A 497 (2003) 535.

[35] R.M. Richardson, Rutherford Appleton Laboratory Report RL-82-035, 1981;

R.J. Newport, J. Penfold, W.G. Williams, Nucl. Instr. and Meth. 224 (1984) 120.

[36] S.F. Mughabghab, Neutron Cross Sections, Academic Press, Orlando, Florida, 1984; http: / /atom.kaeri.re.kr/endfplot.shtml

[37] A.D. Taylor, Rutherford Appleton Laboratory Report RL-84-120, 1984; D.N. Timms, M.J. Cooper, R.S. Holt, P. Postorino, Nucl. Instr. and Meth. A 294 (1990) 509.

[38] A.D. Taylor, B.C. Boland, Z.A. Bowden, Proc. ICANS IX - Int. Collab. on Advanced Neutron Sources, 1986, p. 349 . 
[39] A.D. Taylor, M. Arai, S.M. Bennington, Z.A. Bowden, R. Osborn, K. Andersen, W.G. Stirling, T. Nakane, K. Yamada, D. Welz, Proceedings of the ICANS XI, 1990, p. 705; M. Arai, A.D. Taylor, S.M. Bennington, Z.A. Bowden, R. Osborn, M. Kohgi, K. Ohoyama, T. Nakane, Proceedings of the ICANS XI, 1990, p. 644.

[40] T.G. Perring, A.D. Taylor, R. Osborn, Paul D McK, A.T. Boothroyd, G. Aeppli, Proceedings of the ICANS XII, 1992, p. I-60.
[41] C.K. Loong, L.I. Donley, G.E. Ostrowski, R. Kleb, Proceedings of the ICANS XII, 1992, p. I-36; J.M. Carpenter, C.K, Loong, Proceedings of the ICANS XI, 1990, p. 711; C.K. Loong, J.P. Hammonds, D. Yocum, J.C. Nipko, K. Takeuchi, D. Chen, L.I. Donley, G.E. Ostrowski, R.K. Crawford, H. Belch, R. Kleb, Proceedings of the ICANS XIV, 1998, p. 829.

[42] D. Colognesi, M. Celli, F. Cilloco, R.J. Newport, S.F. Parker, V. Rossi-Albertini, F. Sacchetti, J. Tomkinson, M. Zoppi, Appl. Phys. A 74 (2002) S64. 